GIZMOPOLITAN

\section{O cómo reconciliar la}

\section{femineidad y los videojuegos}

\section{Susana Tosca}

\section{Profesora Titular de Cultura Digital}

Digital Culture/ Mobile Communication Group. Universidad IT de Copenhague. Rued Langgaards Vej 7, 2300 Copenhague (Dinamarca) Email: tosca@itu.dk

\section{Resumen}

Este artículo examina Gizmopolitan, una revista femenina producida por fans cuyo tema central es cómo ser una mujer en Azeroth (el nombre del mundo en que se desarrolla el juego de rol online World of Warcraft). El análisis de esta revista revela interesantes aspectos de la relación dialéctica entre los videojuegos, el género de los jugadores y el contenido producido por fans.

\section{Palabras clave}

Gizmopolitan, world of warcraft, videojuegos, jugadores, género- revistas femeninas

Key Words Gizmopolitan, world of warcraft, computer games, players, gender-women's magazines

\footnotetext{
Abstract

This article examines Gizmopolitan, a fan-produced magazine whose topic is how it is to be a woman in Azeroth (the continent where the MMORPG World of Warcraft takes place). Studying the magazine brings up interesting questions about the dialectics of computer games, player gender and fan produced content.
} 


\section{Introducción}

Gizmopolitan (www.gizmopolitan.co.uk/) es una revista online creada por fans del juego de rol World of Warcraft. Imita el diseño y los contenidos de las revistas femeninas, y su nombre es un guiño a Cosmopolitan, a la que explícitamente pretende imitar.

Gizmopolitan es un objeto mediático interesante por diversos motivos:

- está producida íntegramente por fans

- comenta dos medios al mismo tiempo: videojuego y revistas femeninas
- otorga visibilidad a las mujeres que juegan, un grupo social discriminado dentro y fuera de los videojuegos

¿Qué ocurre cuando un género típicamente femenino migra de plataforma y se convierte en un objeto paródico? Este artículo explora la construcción de la identidad femenina en un mundo tecnológico tradicionalmente tan dominado por hombres como es el de los videojuegos.

\section{Objetivos}

El objetivo de este artículo es, a través del análisis semántico de una revista creada por y para fans femeninas, arrojar luz sobre los mecanismos discursivos que construyen la identidad de jugadora de videojuegos como concepto.

\section{Metodología}

El método utilizado está basado en el análisis de contenidos. Se hace una descripción de los contenidos de la revista (texto e imagen) en relación a tres temas elegidos por su relevancia semántica: el discurso de la revista Cosmopolitan, a la que pretende imitar; su condición de producto hecho por fans y el ethos del juego World of Warcraft, universo-marco de la revista. El análisis posterior se basa en estudios sobre el funcionamiento del modo paródico, en particular las categorías descritas por Harries (2000). La conclusión pone en perspectiva el resultado del análisis en relación a la categoría discursiva de la identidad de las jugadoras de juegos de ordenador. 


\section{Análisis de Gizmopolitan}

Gizmopolitan, que a primera vista pertenece al género de las revistas femeninas, ha lanzado por el momento tres números en la red, y afirman tener 35.000 lectores de 62 países diferentes. No es la única publicación online dedicada al juego World of Warcraft ${ }^{i}$. Como otros juegos en red de nutrida base de jugadores, WoW es objeto de diversas parodias mediáticas que además de revistas y periódicos incluyen películas (machinima), telediarios y otros tipos más clásicos de ficción como cuentos o novelas que desarrollan las historias de los personajes $^{\text {ii }}$. Los habitantes de este enorme universo virtual no se contentan con las (numerosas) horas que pasan jugando y mejorando sus personajes en línea, sino que además emplean mucho tiempo en una labor que aunque derivativa es de una gran creatividad. Mediante la escritura, tratamiento de imagen y video, estos jugadores logran expandir el universo simbólico del juego, que de esta manera cobra nueva vida $y$ logra integrar diversos intereses a partir de una actividad lúdica. El desarrollo de estos productos derivativos también es una manera de participar en la comunidad de jugadores y de obtener así una posición más prestigiosa, que otorga a los autores una categoría de "creadores" por encima de los meros jugadores ${ }^{\mathrm{iii}}$.

Gizmopolitan es "A Lifestyle Magazine for the Women of Azeroth" ${ }^{\text {iv", }}$ y ya a partir de su título y subtítulo tenemos presentes algunas de las contradicciones que son la clave de su particular estilo humorístico. En primer lugar, el título es una combinación de gizmo y Cosmopolitan, la más famosa de todas las revistas femeninas. La primera palabra es difícil de traducir, no tenemos en castellano un equivalente exacto para esta idea, que se refiere a una "cosa" tecnológica e interesante si uno es lo suficientemente nerd. Se aproximan cachivache o cacharro, pero carecen del matiz de objeto deseado y de pequeñez que los gizmos tienen en inglés. Un gizmo es por ejemplo el último modelo de auriculares para un ipod, algo deseable por la novedad y quizá por alguna característica técnica superior, pero en el fondo un poco inútil (dado que todo ipod viene con unos auriculares). Los gizmos son para los nerds, lo que los accesorios (cinturones, pañuelos, joyas, etc.) son para las mujeres (entendida la categoría mujer estereotípicamente, claro está). La presencia de gizmo en una revista femenina indica que a estas mujeres les interesa la tecnología más de lo habitual, que quizá sus accesorios sean tanto de moda como de electrónica. Y esto es muy apropiado para chicas que pasan su tiempo libre jugando a WoW, porque las 4-6 horas diarias que lleva mantenerse a un buen nivel y avanzar su personajes satisfactoriamente no es algo que pueda compaginarse fácilmente con las compras, los tratamientos de belleza y otros requisitos de las mu- 
jeres "a la moda”. De ahí el subtítulo, lifestyle para las mujeres de Azeroth, que aunque parezca que han renunciado a toda esa atmósfera femenina representada por Cosmopolitan al encerrarse casi a tiempo completo en un mundo virtual, todavía tienen los mismos intereses que cualquier otra chica.

¿Se pueden compaginar las identidades de jugadora hardcore y de mujer moderna? Esta es la interesante misión, y podría ser el lema, de esta publicación online, que trata de hermanar las expectativas a menudo opuestas de estas dos identidades ${ }^{\mathrm{v}}$. Si lo consigue o no, hemos de establecerlo a través de un análisis de los tres números disponibles online.

\subsection{Descripción}

Cada número de Gizmopolitan consta de una portada que presenta a una chica de Azeroth bella y aparentemente llena de confianza en sí misma; rodeada por los títulos de los artículos que trae el número, exactamente igual que una revista femenina típica. Los títulos quieren interesar y a veces provocar, pero la portada es esencialmente estática, ya que al ser una publicación online podemos tener siempre todos los títulos de los artículos en un menú a la izquierda y acceder a ellos de esta manera más sencilla.

Una gran diferencia con la revista femenina típica es que no hay anuncios ni secciones en las que se recomienden productos de belleza u otras cosas que comprar. El as- pecto materialista está ausente de la parodia, a pesar de que el comercio con objetos del juego resulta bastante lucrativo para los jugadores de WoW, y los mercados y casas de subasta son una fuente de ingresos importante para muchos.

Si analizamos los artículos (unos 10 por número), podemos distinguir entre los siguientes temas:

- Moda (los mejores looks en diversas categorías, transformaciones, etc.)

- Relaciones amorosas (consejos para flirteo, citas, besos, etc.)

- Etiqueta (bailes, comidas, vacaciones, etc.)

- Hombres deseables (retratos de varios aventureros, a veces con entrevistas y siempre con fotos reveladoras)

- Tests “psicológicos” (por ejemplo: “¿eres una fantasma?”)

Todos estos temas responden a las expectativas de una revista femenina típica, si bien cada número revela al mismo tiempo su filiación lúdica a través de los títulos, declarándose así al mismo tiempo incompatible con una revista tradicional femenina. En el mundo real las mujeres no llevan armadura ("Luscious Leather- Chit explores flattering leather gear for women”), no visitan ciudades virtuales típicas de friquis (“Stormwind Fashion") y por supuesto jamás saldrían con un no-muerto ("Dating a forsaken. Juna explores the pros and cons”). La moda o los bailes no son temas 
especialmente revolucionarios en nuestro mundo real, pero en un juego de ordenador centrado en la violencia y la colaboración en la lucha, el contraste es hilarante. Estas mezclas temáticas son testimonio de la capacidad de las autoras para reflexionar sobre partes diferentes de su identidad (mujer/jugadora), y las contradicciones de esa explosiva mezcla. Nos atrevemos a decir que contrastar ambas identidades de este modo supone un grado de concienciación que debería ser parcialmente liberador.

Veamos un texto típico extraído de un reportaje sobre artefactos mágicos, en que los autores "revelan" noticias sobre artefactos secretos en desarrollo. Se trata por supuesto de artefactos ficticios, que no forman ni formarán parte de los artefactos del juego. El reportaje es una especie de lista de deseos, que revela algunos problemas típicos de la interacción entre jugadores, encarnados en la descripción de un objeto fantástico que pueda eliminarlos. Este es uno de los seis objetos "en desarrollo” (se han mantenido los errores ortográficos del texto original):

\section{Elven Headdress of Underdeveloped Powers of Self-Criticism}

Are you one of those women who know in their heart that they could be leaders - but repeatedly give way to idiots who 'lead' you into disaster after disaster? If so (and we here at Gizmo know the feeling only too well) this is a headdress to die for. How often have you suffered poorly led groups purely because you were intelligent enough to have an understanding of your own weaknesses so you didn't take the lead - while others with little skill or judgment grabbed the power and lead everyone confidently to their deaths?

Wearing this hat will change all that because finally you will have that essential for leadership - naturally underdeveloped powers of self-criticism! Suddenly you will see yourself as 'obviously' the best leader, a 'clearly' superior to the lower life forms you organize into a party!

You will be giving orders to everyone in sight, an ignoring any mistakes with such style that other people will wonder whether it was really a mistake at all! Every woman's dream accessory - we here at Gizmo will be putting in orders for this the moment it hits the streets..."

Este texto es un ejemplo típico del modo conversacional de Gizmopolitan, que se dirige directamente a la lectora, "are you one of those...?", y que busca una identificación en que autoras y lectoras comparten la frustración de tener que soportar a los hombres en general, y a los líderes incompetentes en particular. La mayoría de los artículos comparten este modo discursivo de la queja jocosa, que hace un guiño cómplice a las lectoras y las invita a reírse de su situación de desventaja en la sociedad de Azeroth o de la incomprensión de los hombres. A través del humor se hace una crítica social que a primera vista puede parecer un tanto blanda, ya que no se recomienda ningún cambio, se da a entender 
que las cosas son así y que lo único que podemos hacer es reírnos y aceptarlo (o como en este caso esperar a la llegada de un artefacto mágico que obviamente nunca llegará). A pesar de que muchos títulos son "revolucionarios", como por ejemplo "We are Girls Too, But Will Change For No Man”, el texto de los artículos implica una aceptación de las diferencias, aunque éstas se señalen como injustas: "as a woman in a man's world, we have it hard”. Pero hay mucho más que aceptación silenciosa, como veremos a continuación.

Los artículos de Gizmopolitan apoyan a las jugadoras para que no se avergüencen de su condición de mujeres en un mundo de hombres (el de los videojuegos ${ }^{\text {vii }}$ ). Articular la identidad de jugadora femenina es en sí un reto importante, ya que las mujeres que juegan no sólo tienen que hacer frente a los prejuicios de los jugadores masculinos o del público en general (que las etiqueta como "raras" o antifemeninas), sino también a las propias barreras e inhibiciones, barreras que se manifiestan en una relación ambigua con sus propios deseos. Las mujeres jugadoras han de aprender a aceptarse a sí mismas y a entender los placeres del juego, que no son siempre los que otros esperan de ellas (Taylor, 2003). No sólo les gusta vestir a sus personajes, sino también luchar, colaborar, mejorar de nivel y ganar estatus en el juego. Gizmopolitan anima a sus lectoras a no subordinarse a los deseos masculinos, y utilizar el juego para lo que ellas quieran: "Fight it, girls. Be what you want, and if that's an armour-wearing, sharp weapon wielding maniac, that's fine. Good on you"viii.

Esa es la función más positiva del modo discursivo de Gizmopolitan, el empowerment de las jugadoras de WoW y la toma de conciencia sobre su posición como sujeto lúdico, algo muy necesario en un entorno en que hay gran confusión acerca del rol de jugadora femenina, a pesar de que estudios indican que el porcentaje de jugadoras se acerca al 45\% del total (Mortensen, 2005). Las compañías de juegos quieren atraer al gran mercado femenino, pero sus intentos suelen ser versiones rosa de juegos masculinos (Miss Pac Man), juegos esencialmente estereotípicos (Barbie Fashion Designer) o juegos en los que los personajes femeninos son tan fuertes y hábiles como los masculinos, intentando alcanzar una especie de igualdad lúdica. Mortensen y Corneliussen han demostrado lo absurdo de esta tercera estrategia al hablar de $\mathrm{Ne}$ verwinter Nights (Mortensen 2005) y de cómo la apariencia estereotípica de los personajes significa cosas muy diferentes: los músculos grandes indican poder, las tetas grandes no. La inclusión de mujeres en los juegos, como jugadoras y como personajes, es un paso adelante, pero todavía no podemos hablar de juegos neutrales en cuanto a género. Si examinamos juegos como Neverwinter Nights y WoW entre otros, llenos de personajes femeninos fuertes, hábiles y con cuerpos imposibles, podemos engañarnos acerca de la aparente 
igualdad entre los sexos. La verdadera igualdad está todavía lejos, y Gizmopolitan nos lo recuerda con cada artículo en que se ríe de las exigencias incompatibles de la belleza y la efectividad guerrera. En la superficie, los artículos parecen ser una lista de recomendaciones estéticas, de moda y comportamiento, que perpetúan los estereotipos, pero si leemos los textos con atención detectamos un sarcasmo acerado que revela la contradicción de los mundos virtuales actuales, que no hacen sino reproducir las contradicciones de nuestro mundo real. Como Mortensen y Corneliussen observan irónicamente:

But at the same time it is perhaps one of the most perfect representations of the modern feminist trap. The female characters in $N W N$ do everything the boys do, and they still manage to look feminine while doing it. So rather than being offered freedom from female stereotypes, the game just adds the demands to a male to the demands to a female. It sounds almost social realistic, and who would have thought that about a fantasy role playing game. (Mortensen, 2005: 15)

Gizmopolitan transmite el mismo mensaje, con una gran dosis de humor y ofreciendo modelos alternativos de comportamiento. Estos modelos alternativos no son como podría suponerse únicos. No hay un mensaje monolítico en que se recomiende a las lectoras no preocuparse por la apariencia y dedicarse a la destrucción y a la guerra sin reparos, como la cita arriba podría indicar.
Se ofrecen modelos diferentes que conviven entre sí: la guerrera pura y dura, la jugadora a quien sólo le interesa la moda, la que le gusta jugar con los estereotipos y burlarse de los hombres, o la enamorada permanente. El mensaje es que cualquiera de ellos es aceptable; es más, que las jugadoras de WoW varían cíclicamente de preferencias y objetivos. No hay una sola forma de disfrutar de este juego, sino que las jugadoras han de hacer lo que más les guste a ellas, y no actuar para dar gusto a otros. La única obligación es divertirse. Y lo interesante, como veremos más abajo, es que todos estos modelos surgen en cierta manera contra el ethos del juego.

Las ilustraciones que acompañan los diversos artículos refuerzan este mensaje principal. Se trata de instantáneas tomadas dentro del juego (y no dibujadas para la ocasión), lo que otorga a la revista un valor de documento y además implica que las reporteras han recorrido Azeroth a la búsqueda de modelos y situaciones para retratar. Muchas de las fotos presentan a los personajes en posturas paródicas y burlonas, que resultarán divertidas para los iniciados (los otros jugadores). El juego tiene una serie de emoticones pre-definidos, o gestos disponibles para todos los personajes, como por ejemplo: llorar, arrodillarse, pedir, reír, saludar, etc. Hacer click en uno de ellos conlleva perder momentáneamente el control del personaje, que procederá a realizar el gesto elegido en una manera bastante robótica y estereotipada (puesto 
que todos los personajes lo hacen del mismo modo, independientemente de su raza, sexo o complexión física).

Los jugadores de WoW pueden decodificar la naturaleza a menudo carnavalesca de estas imágenes, que incluso en las poses estáticas de "modelos" que muestran ropa subvierten la naturaleza del juego, diseñado para que los personajes estén siempre en movimiento, luchando y obteniendo puntos. Utilizar Azeroth como un estudio fotográfico, o un set de cine como se hace en machinima, es una apropiación del juego para los propios fines críticos y creativos que lo desposee temporalmente de su condición de juego para convertirlo en un instrumento de comunicación. Los jugadores pasan de ser objetos a sujetos, y este movimiento es compartido con los lectores de Gizmopolitan, que pueden disfrutar de estas imágenes "fuera de contexto".

Esperamos haber demostrado suficientemente la condición múltiple de nuestro objeto de estudio. Ahora procederemos con nuestro análisis observando como Gizmopolitan es y no es al mismo tiempo todas las cosas que juega a ser: una revista femenina, un producto del juego WoW, y un producto derivativo de cultura de fans. A través de la comparación y el contraste con estos objetos relacionados podremos profundizar aún más en la clave de su condición especial.

\section{2. ¿Una copia fiel de Cosmopolitan?}

Es inevitable preguntarnos si Gizmopolitan se parece realmente a la revista con la que se identifica a través de su nombre. Cosmopolitan es la revista femenina de más éxito en el mundo; según su editorial, Hearst Magazines, tiene 58 ediciones internacionales y se distribuye en más de 100 países, con millones de lectoras.

En el mundo de las revistas femeninas, Cosmopolitan ocupa una posición especial debido a su enfoque sexual, mientras que otras revistas femeninas dirigidas al mismo público, como Vogue o Elle están más dedicadas al mundo de la moda. Cosmopolitan, que nació como una revista para la familia, se convirtió en 1965 de la mano de Helen Gurley Brown en el foro que confirmaba que las mujeres tenían derecho a tener vidas sexuales plenas independientemente del matrimonio. Los artículos sobre sexo, citas y relaciones con los hombres ocupan la mayor parte del espacio de la revista (aparte de la publicidad), y la filosofía de la publicación es la de animar a las mujeres jóvenes a tomar las riendas de su propia vida y a ser independientes económica y emocionalmente, expresado en el lema: "For Fun, Fearless Females". Sin embargo, y aunque este no es el lugar para hacer un análisis de Cosmopolitan, una lectura más detallada revela que las "lectoras sin miedo" parecen bastante acomplejadas, dado el grado de espacio dedicado a 
convencerlas de que su cuerpo y sus habilidades son aceptables.

Parece que Cosmopolitan y Gizmopolitan comparten la filosofía de que el matrimonio no es el único camino para las chicas modernas, "A marriage is nothing more than a name, that bind you to him. Be free instead and bind the men you like to you for as long as you want and no more. Go out with them, find out the things you like and argue about the things you don't. Remember - marriage isn't a must!" Pero si una escarba bajo la superficie de las múltiples citas y los catálogos de posturas sexuales de Cosmopolitan, encontrará un mensaje bastante más conservador. Keller Simon hace el siguiente comentario:

Cosmopolitan is a contemporary female bildungsroman, an illustrated, commercialized, and fragmented woman's coming-of-age novel in which the reader herself functions as heroine, making her way selectively through the typical crises of late adolescence and early adulthood that the text presents to her. It is also a romance, a love story melodramatically simplified and erotically idealized, marked by a descent into a dream world. And it is a novel of manners, devoted to the translation of social gesture, dress, look, and public behavior into explicit meanning and concerned with the individual's relationship to social convention (Keller Simon 1999: 117)

En Cosmopolitan, como observa Keller Simon, lo más importante es la relación con los hombres, o deberíamos decir con “el hombre”. Y se podría considerar que las otras secciones están subordinadas a este tema: ropa o maquillaje son las armas de las jóvenes modernas para encontrar marido. Y aunque las historias son totalmente fantásticas (también un análisis de las imágenes revelará un mundo "of impossibly flawless beauty"), la revista incluye la contradicción de las cartas de las lectoras, que son siempre de chicas desesperadas, inseguras, que dudan acerca de su imagen, de cómo comportarse, y que incluso son psicológicamente inestables. Cosmopolitan incluye además siempre anuncios que indican que las lectoras no responden a ese ideal de mujer fieramente independiente, segura de sí misma y encantada con su vida sexual, con los productos para dietas drásticas, cirugía estética y lubricadores vaginales (edición US).

En este sentido, las similitudes entre Gizmopolitan y Cosmopolitan son sólo superficiales, ya que el principal tema de Gizmopolitan no es el ayudar a las jugadoras a encontrar pareja en el juego. Aunque los títulos de los artículos podrían intercambiarse en ocasiones, ya hemos visto que el enfoque de Gizmopolitan es la exploración de la identidad de jugadora femenina, y no la subordinación a un ideal masculino. Los testimonios de las lectoras de Gizmopolitan no revelan las mismas contradicciones que Keller Simon encuentra en Cosmopolitan. Las jugadoras entrevistadas en los artículos son siempre conscientes de la dualidad de su posición como mujeres/jugadoras, y 
capaces de reírse de sus problemas igual que las redactoras de la revista. No hay tragedia en Gizmopolitan.

Pero Cosmopolitan no es sólo una guía de conducta sexual. A pesar de las contradicciones esbozadas, la revista hace uso de un modo discursivo propio y muy especial que David Machin llama el "problem-solution schema”. Cualquier asunto, sea afectivo, de belleza o laboral, se presenta como un problema que la chica Cosmo puede solucionar si se aplica, y para cuya solución siempre se dan ideas y consejos. Si bien esto puede resultar peligroso porque transmite toda la responsabilidad sobre desigualdades sociales al individuo (que si fracasa lo habrá merecido), tiene un innegable aspecto empowering, y empuja a las lectoras a actuar y no conformarse con las situaciones desventajosas. Este tipo de discurso no se da en Gizmopolitan, que como hemos visto alienta más la burla y la aceptación humorística de las desigualdades. Sin embargo ambas publicaciones se parecen en su afán revolucionario, algo que no deberíamos despreciar a pesar de todas las contradicciones y estereotipos que Cosmopolitan representa. Como dice Machin en su análisis de varias ediciones de la revista en diversas lenguas:

Cosmopolitan has consistently, and globally, propagated its ideal of the 'fun, fearless female'--a woman who has a career and remains independent, although she is frequently involved in pleasure-seeking casual affairs. In many cultural contexts this ideal is just as revolutionary (...) For women it holds out the prospect of liberation from patriarchal relations; for the traditional, patriarchal societies in which they live in threatens the fabric that held them together (Machin, 2007)

Si consideramos el mundo de los videojuegos como esencialmente patriarcal y masculino, la burla juguetona de Gizmopolitan puede tener el mismo efecto liberador que Machin le atribuye a Cosmopolitan en sociedades más tradicionales que la nuestra.

\section{3. ¿Parte del juego?}

Hemos insinuado más arriba que Gizmopolitan iba contra el ethos de WoW, porque la esencia del juego es desarrollar nuestros personajes acumulando puntos para subir de nivel, y esto sucede mediante el combate, la recolección de artículos diversos y la realización de misiones o quests. La interacción social con otros jugadores es importante para cumplir determinadas misiones, sobre todo cuando se sube de nivel, pero también es posible llegar a niveles altos sin tener demasiada relación con otros o agruparse en clanes estables. En este sentido, los intereses que fomenta Gizmopolitan: la moda, las relaciones con otros jugadores o la etiqueta, no son relevantes si consideramos WoW exclusivamente como videojuego. Incluso son perniciosos ya que distraen de la actividad principal y loable de obtener puntos y subir los personajes de nivel. 
Pero en realidad lo que demuestra Gizmopolitan (junto con otras muchas actividades que surgen alrededor de los videojuegos ${ }^{\mathrm{x}}$ ) es que entender los videojuegos como actividades exclusivamente formales y desprovistas de interés social más allá que el cumplimiento de unas determinadas reglas de interacción es una postura extremadamente reduccionista. Peor aún, una actitud excesivamente formalista nos impide entender los videojuegos como objetos culturales: WoW es mucho más que un juego. Es un mundo en el que los jugadores desarrollan relaciones sociales bastante sofisticadas y dedican mucho tiempo a actividades que no son estrictamente "del juego", ya que no otorgan puntos ni representan ninguna ventaja en cuanto a la mejora de los personajes. Al contrario, como estudia en profundidad TL Taylor (2006), hay evidencia de que la cultura que surge en torno a los universos lúdicos de los juegos de rol online se asemeja a cualquier comunidad humana en la complejidad de sus relaciones y el grado de implicación de los participantes.

Por tanto, actividades como las recogidas en Gizmopolitan (interesarse por la moda y dedicar energías a buscar un determinado sombrero aunque no otorgue mejores estadísticas vitales) serían testimonio de que la interacción social en $\mathrm{WoW}$ se rige por otros valores que los meramente relacionados con las reglas del juego. En nuestro artículo sobre la importancia de la moda en el universo de WoW (Klastrup y
Tosca 2009) demostramos que comunicación simbólica a través del vestido tiene la misma importancia para los jugadores ${ }^{\mathrm{xi}}$ en el mundo virtual que el real. Esto apunta a que el universo de los videojuegos no es tan limitado como piensan los que nunca se han acercado a él, y que las relaciones sociales y comunicativas que surgen en torno a los juegos no tienen nada que envidiar a las de otros contextos de agrupación humana.

Como ilustración de todo esto describiré un ejemplo tomado de Gizmopolitan, en que los lectores responden directamente al contenido de los artículos, demostrando que la comunidad de jugadores se interesa por algo más que por acumular puntos ciegamente. En el primer número de la revista apareció un artículo titulado "Five things to know about dating a Paladin", en que las redactoras daban consejos a las lectoras acerca de cómo afrontar una relación amorosa con un “paladín”. El paladín es una de las clases que los personajes de WoW pueden elegir, y se caracterizan por ser la encarnación del caballero medieval a mitad de camino entre un guerrero y un clérigo (otras dos clases "puras" del juego). Este tipo de personajes es capaz de luchar y curar, maneja cualquier arma, y suele estar muy apegado a su fantástica armadura, ya que depende de ella para mejorar sus habilidades. Es un personaje familiar en la literatura fantástica, y suele regirse por un estricto código de honor (que a veces incluye la castidad) y que le hace un compa- 
ñero de grupo difícil cuando hay que utilizar métodos poco ortodoxos.

El artículo describía en clave de humor los problemas de enamorarse de un hombre tan estricto y entregado a su causa, y le retrataba como excesivamente apegado a su armadura, a su espada y a su caballo, incapaz de concentrar su atención en una mujer:

"Well, if you want to go for a romantic date, don't choose a spot close to the water or a lake. Even a small moon is enough to reflect the armour in the water, so they will look more at that than at you" ${ }^{x i i}$.

Este artículo tuvo gran impacto en la comunidad de jugadores, que inundaron la redacción con emails felicitando a las autoras, y también protestando en contra de los prejuicios de la descripción de los paladines. Para reparar la injusticia, un grupo de paladines decidió afrontar una de las misiones más peligrosas del juego (Molten Core) parcialmente desnudos, demostrando así que no es cierto que los paladines amen su armadura sobre todas las cosas. Estos jugadores enviaron una serie de fotografías a la redacción, que fueron publicadas en un artículo titulado "Topless Paladin Run" en el número 2 de la revista:
Gráfico n ${ }^{\circ}$ 1: Topless Paladin Run

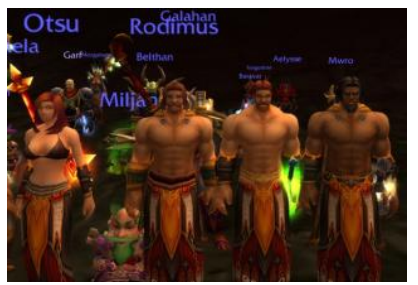

Fuente: Gizmopolitan

Este episodio evidencia un diálogo entre Gizmopolitan y la comunidad de jugadores, que va más allá de las lectoras femeninas, y demuestra que los jugadores en general (en este caso paladines, hombres) están abiertos a la reflexión cómica sobre su propio papel en el juego y su identidad (fantástica pero también real). La mayoría de los jugadores de WoW son algo más que máquinas de hacer puntos, y están dispuestos a participar en actividades sociales que les "distraigan" de su ocupación guerrera primordial. Así podemos afirmar que aunque Gizmopolitan va en contra del ethos de WoW exclusivamente entendido como las reglas del juego, en realidad contribuye a demostrar que el juego es mucho más que sus reglas, ya que la interacción simbólica es algo que sus jugadores valoran mucho.

\section{4. ¿Típica producción de fans?}

El último ángulo desde el que observar Gizmopolitan tiene que ver con su condición de producto creado por fans. La producción típica de los fans de videojuegos puede dividirse en los objetos que estrictamente se refieren al juego (walkthroughs, 
guías de estrategia, skins o programas que mejoran las capacidades de los jugadores), y objetos que expanden el universo ficticio del juego pero no tienen repercusión en el propio acto de jugar (relatos de ficción, dibujos, poesías, películas y parodias mediáticas como telediarios). Gizmopolitan pertenece al segundo grupo, y es testimonio de que la vida cultural que surge alrededor de los videojuegos es mucho más rica de lo que se suele suponer. Como destacábamos en el apartado anterior, este tipo de divertimentos no tiene ninguna consecuencia ni ventaja en el mundo del juego, pero les proporciona a los jugadores un foro en el que sumergirse en el universo fantástico de Azeroth y contrastar la actividad jugadora con otros intereses "externos" que la ponen en perspectiva y la integran aún más en su vida.

Hay otros ejemplos del interés por la moda de los jugadores de videojuegos, pero suelen ser más instrumentales, como en Los Sims o Second Life, en que los jugadores crean skins, o identidades visuales para personajes y las intercambian online.

Como recoge Hannah Wirman (2008), se trata también de una actividad principalmente femenina, pero en estos skin jobs podemos casi hablar de "artesanía", ya que lo que se valora es la habilidad técnica que luego se aplicará directamente en el juego. En cambio, Gizmopolitan es exclusivamente un comentario desprovisto de toda utilidad o aplicación directa en el juego. El auténtico valor de Gizmopolitan, igual que otras parodias en machinima, es que expande el universo del juego y lo convierte en un objeto aún más importante y más integrado en la realidad de los jugadores. Ondrejka habla del poder de la creatividad de los jugadores "to maximize the virtual world's growth in order to build an online space as rich and complex as the real world" (2006: 1). Es una idea muy ambiciosa, que sin embargo ilustra el poder simbólico de esta actividad creativa.

\section{La naturaleza de la parodia en Gizmopolitan}

La parodia tiene una larga y productiva historia en la literatura y cine occidentales, incluso en artes plásticas o en música. Grandes obras como El Quijote nacieron como parodias. A pesar de ello, ha habido una tendencia a despreciar la parodia como un género parásito e inútil, aunque el nivel metadiscursivo es omnipresente en nuestros tiempos postmodernos (Hutcheon, 1985: 1). Si consideramos que la intertextualidad es una característica fundamental y casi inevitable de la narración, es casi inevitable encontrar elementos paródicos en la remediación ${ }^{\text {xiii }}$ de géneros. La parodia, por 
otra parte, requiere un esfuerzo interpretativo por parte del lector:

Both irony and parody confuse the normal processes of communication by offering more than one message to be decoded by the reader, which may also serve to conceal the author's intended meaning from immediate interpretation (Rose, 1979: 51)

Esta doblez es la característica más señalada de Gizmopolitan, y funciona no sólo al nivel de cada frase o expresión, sino también como marco interpretativo general. Gizmopolitan es una parodia de dos géneros al mismo tiempo: la revista femenina y el juego de rol online. La revista femenina, a pesar de su colorido y su afán por resultar ligera y divertida, carece de humor, y se toma a sí misma y a sus lectoras muy en serio. No puede hacer otra cosa, ya que depende de que las lectoras puedan identificarse con la lectora modelo que se construye alrededor de temas tan vitales y delicados como la propia identidad y sexualidad. Gizmopolitan por el contrario se ríe explícitamente de (y con) sus lectoras. Los consejos van acompañados de comentarios irónicos que dan a entender que no son consejos reales, sino imitaciones de los consejos que se suelen dar en las revistas femeninas. Las redactoras tienen un público cómplice, que sabe que la posibilidad de aplicar tales consejos en el mundo guerrero de WoW es muy limitada, por no decir imposible.

En cuanto al juego de rol, Gizmopolitan lo parodia ignorando por completo el hecho de que es un videojuego construido para que los jugadores pasen el tiempo ganando puntos. Como hemos analizado más arriba, la revista se concentra exclusivamente en las relaciones sociales y amorosas que no pertenecen al juego y al sistema de reglas. Es como si las redactoras afirmaran: nosotros usamos el juego para otra cosa. Tienen su propia agenda y no es la misma que la de los creadores del juego o los jugadores más acérrimos.

Gizmopolitan niega por tanto al mismo tiempo la seriedad de las revistas femeninas y la obsesión por la obtención de puntos de los juegos de rol. Les roba su razón de ser a ambos objetos, y afirma su derecho a apropiarse de ellos para sus propios fines. Este es el modo más radical de parodia que pueda imaginarse, ya que como advierte Hutcheon, a menudo el objetivo de la parodia no es el texto original o parodiado (1985: 51), sino que el objetivo es el comentario o la sátira social. En Gizmopolitan, la intención de la sátira está dirigida a desmontar los prejuicios habituales contra las mujeres que se atreven a jugar a videojuegos (y que se divierten con ello). Precisamente porque estos prejuicios vienen desde ambos bandos: los jugadores masculinos que las ven como "intrusas", y las mujeres que no juegan, que las ven como "bichos raros", Gizmopolitan hace uso de una doble parodia que desestabiliza los dos ámbitos.

Dan Harries ha investigado la "pragmática de la parodia” (2000), y describe los méto- 
dos y técnicas que emplea el modo paródico, y como afecta a sus receptores. Gizmopolitan emplea los seis métodos que enumera Harries (2000: 37-39):

- Reiteration. Se trata de una cita o evocación del original, como por ejemplo en el modo en que se diseñan las portadas de Gizmopolitan, respetando las convenciones de las revistas femeninas pero ya introduciendo los elementos ajenos: el rostro de personajes del juego.

- Inversion. Se utilizan expresiones o temas en sentido opuesto al original. Por ejemplo un artículo con consejos acerca de cómo encontrar un hombre, además de contener recomendaciones sobre como atraerlos también incluye cómo capturarlos a martillazos y llevárselos a casa para divertirse una tarde ${ }^{\text {xiv }}$.

- Misdirection. Un giro inesperado en medio de una escena típica. Por ejemplo, la sección de anuncios para buscar novio se convierte en una especie de confesión humorística sobre la propia personalidad $^{\mathrm{xv}}$, lo que rompe totalmente las expectativas del género "anuncios personales".

- Literalization. Hacer evidentes ciertas convenciones. Por ejemplo el modo burlón y estereotípico en que se "fotografía” a los hombres semidesnudos en la sección "Men on our lust list".

- Extraneous inclusion. Introducir un elemento extraño y ajeno al universo de la ficción. Por ejemplo, un reportaje de moda y vestidos bonitos incluye también recomendaciones sobre armadura y cuando es apropiado quitarse las ropas de combate para acudir a una fiesta.

- Exaggeration. Exageración. Este es un modo discursivo constantemente presente en los textos de la revista, como es evidente en los fragmentos reproducidos hasta ahora.

Mucho se ha escrito sobre el poder liberador de la parodia, y sobre cómo puede llegar a perder su fuerza si degenera en entretenimiento inane. Sin embargo, aunque no podamos decir que este tipo de parodia desestabilice el sistema cultural o libere a los que la practican, sí creemos que "questions the legitimacy of established social norms" (Harries, 124), ya que logra poner de relieve las múltiples contradicciones de la identidad de las mujeres que disfrutan de los videojuegos, y de las convenciones sociales (reales y virtuales) que las oprimen. 


\section{Conclusión: la expresión de la feminidad en el ámbito de los videojuegos}

La presencia de las mujeres en el ámbito de los videojuegos ha sido objeto de discusión y debate desde principios de los noventa ${ }^{\mathrm{xvi}}$. A pesar del crecimiento continuo del porcentaje femenino en el total de jugadores, especialmente en espacios domésticos, la zona cultural de los videojuegos se sigue percibiendo como eminentemente masculina. Como lo expresan Bryce y Rutter, la actividad "is subject to the gender dynamics of technological access and the stereotyping of associated skills and interests in important ways" (2006: 200)

Como se hace evidente en el trabajo empírico y teórico ${ }^{\mathrm{xvii}}$, la identidad de "jugadora" es incluso más problemática que la de "jugador”. Los videojuegos conllevan un gran estigma social, y el discurso institucional y mediático acerca de los hombres y chicos que juegan suele ser alarmista y lleno de prejuicios, centrado en temas como la violencia o el carácter antisocial de la actividad. Las mujeres y chicas que juegan han de añadir a lo anterior el estereotipo de raras, de mujeres masculinizadas por interesarse por esta actividad. Si bien está aceptado un cierto interés social en algunos juegos (como utilizar la consola wii en una fiesta), la actividad jugadora constante que requiere un juego como WoW (varias horas diarias) se precibe como problemáti- ca en el caso de las mujeres, como un indicador de que algo no es normal.

En ese sentido Gizmopolitan llama la atención sobre lo absurdo de estos prejuicios, insistiendo en que la actividad lúdica es un pasatiempo tan loable como cualquier otro entretenimiento, divertido y sobre todo, extremadamente social. Y no se trata únicamente de insistir en que las chicas también pueden jugar, sino también de defender que los videojuegos pueden entenderse de formas diversas. Gizmopolitan es el antídoto contra la concepción totalmente hardcore de los juegos de rol, en que lo único importante es obtener puntos sin detenerse a mirar el paisaje o hablar con los demás personajes.

Si entendemos que el género es una categoría social (y el sexo biológico), y que la renegociamos constantemente en nuestra vida diaria (Bryce y Rutter, 2006: 187), Gizmopolitan es una especie de manifiesto que proclama que la identidad de jugadora no puede encuadrarse en la estrechísima zona que habitualmente se le otorga. Gizmopolitan reivindica una identidad múltiple que contiene elementos tanto del universo del juego como de lo que significa ser mujer en la vida real. Sus armas son la parodia y el humor, expresados de un modo tan creativo y agudo, que logran una auto-afirmación mucho más efectiva que la 
queja amarga. Ellas sí que son "fun, fearless females”, y si alguien no está de acuerdo, se le cose a espadazos.

\section{Referencias}

BOLTER, J.D. y GRUSIN, R. (1999): Remediation: Understanding New Media, Cambridge, MA: MITPress.

BRYCE, J. y RUTTER, J. (2002): “Killing Like a Girl: Gendered Gaming and Girl Gamers' Visibility”. In Mayra, Frans (ed.). CGDC Conference Proceedings. Tampere: Tampere University Press.

BRYCE, J. y RUTTER, J. (eds.) (2006): Understanding Digital Games. London: SAGE.

CASSELL, J. y JENKINS, H. (1998): From Barbie to Mortal Kombat. Gender and Computer Games. Cambridge, MA: The MIT Press.

EGENFELT-NIELSEN, S.; HEIDE-SMITH, J. y TOSCA, S. (2008): Understanding Videogames. London: Routledge.

HARRIES, D. (2000): Film Parody. London: BFI Publishing.

HUTCHEON, L. (2000): A Theory of Parody. The Teachings of Twentieth Century Art Forms. Chicago: University of Illinois Press.

KAFAI, Y. B. et al. (2008). Beyond Barbie and Mortal Kombat. New Perspectives on Gender and Gaming. Cambridge MA: The MIT Press.

KELLER S., RICHARD (1999): Trash Culture. Popular Culture and the Great Tradition. Berkeley: University of California Press.

KERR, A. (2003): "Girls Women Just Want to Have Fun - A Study of Adult Female Players of Digital Games”. In Copier, Marinka (ed). Level-Up. Digital Games Research Conference. Utrecht: Utrecht University.
KLASTRUP, L. y TOSCA, S. (2009) (forthcoming). "Because it just looks cool!" Fashion as character performance: The Case of $W_{0} W$ ”. Journal of Virtual Worlds Research.

MACHIN, D. y VAN LEEUVEN, T. (2007): Global Media Discourse. London: Routledge.

MORTENSEN, T. y CORNELIUSSEN, H. (2005): "The Non-sense of Gender in Neverwinter Nights", in Women in Games (2005) Conference papers, Dundee, Scotland 8-10 August 2005.

ONDREJKA, C. R. (2006): Escaping the Gilded Cage: User Created Content and Building the Metaverse. New York Law School Law Review.

ROSE, M. A. (1979): Parody / Meta-fiction. An Analysis of Parody as a Critical Mirror to the Writing and Reception of Fiction. London: Croom Helm.

TAYLOR, TL. (2003): "Multiple Pleasures: Women and Online Gaming," Convergence, Vol. 9, No.1, 21-46, Spring 2003.

TAYLOR, TL. (2006): Play Between Worlds: Exploring Online Game Culture. Cambridge, MA: The MIT Press.

WILlIAMS, Dmitri; Yee, Nick; E. Caplan, Scott. 2008 "Who plays, how much, and why? Debunking the stereotypical gamer profile". Journal of Computer-Mediated Communication, VL: 13, NO: 4

WIRMAN, H. (2008): Virtual Threads of a Skin. Women Weavers and the Practice of Skinning The Sims. Proceedings of The Player Conference. Copenhagen: IT University of Copenhagen, August, 2008.

Cita de este artículo

TOSCA, S. (2011) Gizmopolitan. 0 cómo reconciliar la femineidad y los videojuegos. Revista Icono14 [en línea] 1 de enero de 2011, Año 9, Volumen 1. pp. 03-20. Recuperado (Fecha de acceso), de http://www.icono14.net 
${ }^{\mathrm{i}}$ Este es un juego de rol online en que los jugadores han de desarrollar sus personajes mediante la lucha y el aprendizaje de diversas "profesiones". World of Warcraft sucede en un mundo de fantasía poblado por dos facciones enemigas en que humanos, elfos, enanos, orcos y otras razas "clásicas” se enfrentan por la supremacía absoluta.

${ }^{i i}$ Por ejemplo hay periódicos (http://www.theallianceherald.co.uk/), canales de noticias y películas en el mundo del juego (www.machinima.com o www.warcraftcinema.com) o archivos de literatura creada a partir de los personajes del juego (http://www.fanfiction.net/game/Warcraft/).

iii Esto a veces se hace explícito, como en Gizmopolitan, donde el primer número tiene una sección llamada "On the Pipeline. Articles we might write if we get enough egoboo".

${ }^{\mathrm{iv}}$ El nombre del mundo del juego donde se desarrolla World of Warcraft.

${ }^{v}$ Por desgracia, Blizzard, la compañía que publica World of Warcraft, no ofrece ningún dato acerca de sus jugadores, pero estudios estadísticos previos en otros juegos similares (como por ejemplo el de Williams et.al. para Everquest2 de 2008) indican que la distribución de género es $80 \%$ jugadores masculinos, $20 \%$ femeninos. Si tenemos en cuenta que el juego tenía 12 millones de jugadores a finales de 2008, obtendremos una cifra nada desdeñable de 2,4 millones de mujeres.

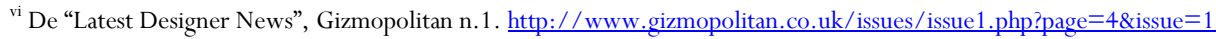

${ }^{\text {vii }}$ Hay bastantes textos en torno a los conflictos que surgen cuando las mujeres entran en el mundo de los videojuegos, ya sea como jugadoras o diseñadoras. Un reciente esfuerzo recopilador ha dado lugar al notable "Beyond Barbie and Mortal Kombat New Perspectives on Gender and Gaming”, editado por Kafai et.al. 2008.

viii De “We are Girls Too, But Will Change For No Man” (http://www.gizmopolitan.co.uk/issues/issue3.php? page $=12$ \&issue $=3$ )

${ }^{\text {ix }}$ Del artículo "Five things to know about dating a paladin" (http://www.gizmopolitan.co.uk/issues/issue1.php? page $=3 \&$ issue $=1$ )

${ }^{\mathrm{x}}$ Véase cap. 7 de Egenfeldt-Nielsen, Heide-Smith y Tosca. 2008.

${ }^{\mathrm{xi}}$ Decimos jugadores porque en nuestra encuesta obtuvimos los mismos resultados para mujeres que para hombres, con lo que podemos demostrar que la comunicación simbólica no es dominio exclusivo de las mujeres.

${ }^{\text {xii }}$ Del artículo "Five things to know about dating a paladin" (http://www.gizmopolitan.co.uk/issues/issue1.php?page=3 $\underline{\text { \&issue }=1)}$

xiii En el sentido de Bolter/Grusin, 1999.

xiv “You're pretty much ready to go out there and get that man! Make sure to take a club-like weapon, I suggest a club, you'll need to use this when the man turns his back, just smash him in the back of the head (it may take several hard blows for certain men to fall to their knees, this is where your aerobics and boxercise training will pay off) and he's yours for the evening or for as long as you wish to keep your man captive". http:/ / www.gizmopolitan.co.uk $/$ issues $/$ issue3.php?page=7\&issue=3

xv "Desperate editor seeks boyfriend to stave off match-makin friends, anything considered, intelligent confident arm candy who can make a girl laff preferred, long hair and exciting sinister streak = irresistible. Replies please from guys fitting this description who like a girl with unconventional looks, a sparky personality and a demanding job. Failin that, replies from anybody who promises not to lick my leg in public. Reply to Box 601, Earthen Ring." Gizmopolitan, issue 2.

http: / / www.gizmopolitan.co.uk/issues/issue2.php?page $=12$ \&issue $=2$

${ }^{\text {xvi } V e ́ a n s e ~ l o s ~ t e x t o s ~ r e c o p i l a t o r i o s ~ d e ~ C a s s e l l ~(1998), ~ B r y c e / R u t t e r ~(2002), ~ K e r r ~(2003) ~ o ~ T a y l o r ~(2003) ~}$

xvii Íbid. 